\title{
Influence of M2M Solution on Multimedia Communication Technology Development
}

\author{
Chaoyan $\mathrm{Wu}^{1, \mathrm{a}}$ \\ ${ }^{1}$ Urban Research Institute, Sichuan University, Sichuan, 610064, China \\ aanprosia2013@126.com
}

Keywords: Multimedia communication technology, M2M solution, Collaborative development

\begin{abstract}
The application of M2M solution has been a focus in the multimedia communication technology development in many fields such as electric power, transportation, safety and environmental protection and so on. The goal of this paper is to interpret the development process of multimedia communication technology from a M2M solution perspective, and grasp the nature of change and have more comprehensive understanding influence of M2M solution on development of multimedia communication technology through analyzing relationship between M2M solution and communication technology industry.
\end{abstract}

\section{Introduction}

With the emergence of Web3.0, credible SNS (social network service system), manageable VoIP (Voice over Internet Protocol) and IM (Instant Messaging) and controllable Blog or Wiki developing rapidly. These trends of integration of digital communication and information processing effectively lead to the combination of media content and the business intelligence and melt of communication and management ${ }^{[1]}$. A new generation of digital communication technology is M2M solution, which achieves the communication between machine and machine. As shown in Figure 1, it can be machine to machine, machine to man, man to mobile terminals, and mobile terminals to machine. M2M solution will connect machinery and equipment into a network which all over human's daily life with integrate communication and network. This technology will make these multimedia communication devices become more intelligent, allow mankind to create rich applications and bring about a new round of changes to the daily life and industrial production.

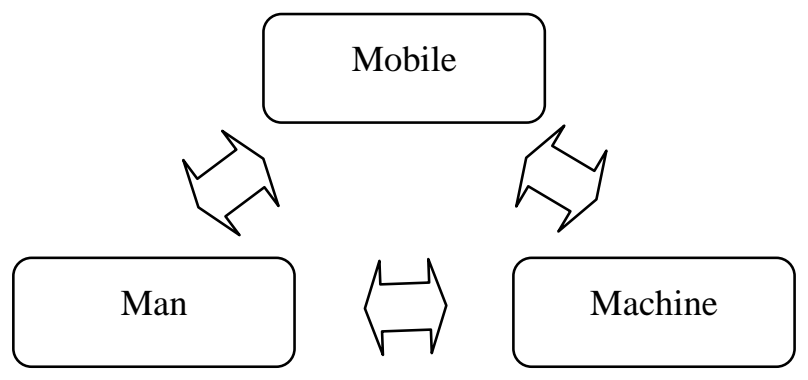

Fig. 1 M2M schematic diagram

A characteristic of the new generation network is making the object of multimedia communication services extend from people to any one thing, and then the network technology will be omnipresent. Computer and communications equipment will cover all areas, the communication mode not just in the relationship between people and people, but also exist between people and machine, machine and machine. The equipment or appliances which can connect the Internet is much more than now, which are from the refrigerator to the MP3 player, from the vehicle to the engineering equipment control system, form the electronic vending machine to the street light, they work as the Internet computer, forming machine to machine (M2M) communication mode. With the expansion of the sensing technology progress and wireless network bandwidth, the application of machine to machine which is still in the early stages will become the driving force for relationship between enterprise and individual which belong to the communication industry [2]. 


\section{The influence of M2M solution on multimedia communication technology form}

Multimedia communication technology from has experienced two transitions. The first transition has two characteristics: First, the modern significance of the communication institutes appeared in succession and promoted the development of the multimedia communication technology industry to become an independent industry; the second is multimedia communication technology industry's core business focus on modern digital communication technology. Background of second major communication technology industry transition and upgrading is the new demand of M2M solution with rising digital communication technology. The European M2M market has been relatively matures, which forming a complete industrial chain. Especially in Western Europe market, it has realized the application of safety monitoring, mechanical services, vehicle information communication terminal, vending machines, public transportation systems, fleet management, industrial process automation, information and other areas of the city.

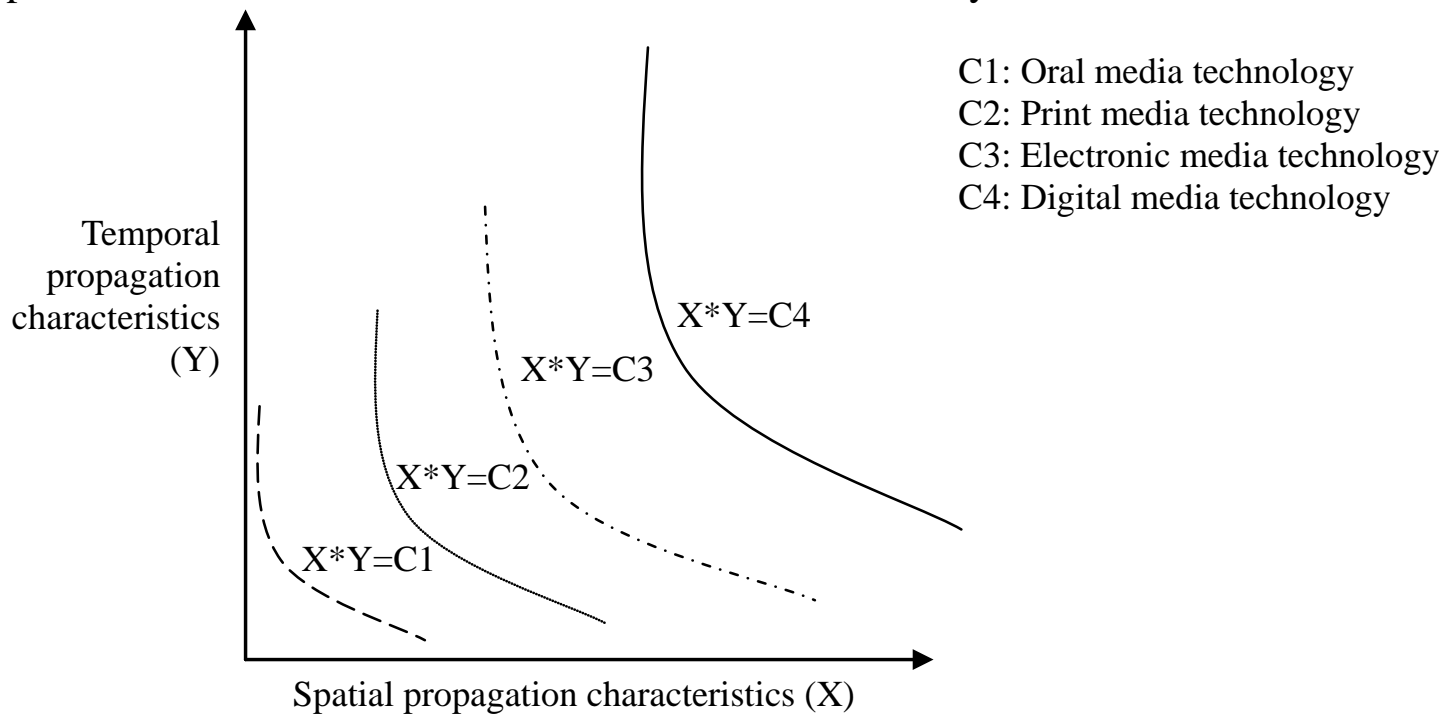

Fig. 2 Spatial and temporal characteristics of communication technology industry

In the context of M2M solution, the multimedia communication technology can be measured by temporal characteristics and spatial characteristics of communication media [3]. The continuous progress of spatial and temporal properties contributes to the media technology development, while the development is not continuous change, which has obvious technological characteristics of times. The temporal and spatial alternation of media can use Figure 2 to explain. The spatial and temporal characteristics function of media propagation is $\mathrm{XY}=\mathrm{C}$, where $\mathrm{X}$ is the parameters of spatial propagation characteristics, $\mathrm{Y}$ is the parameters of temporal propagation characteristics. When the parameter of temporal propagation characteristics is strong, the parameter of spatial propagation characteristics is relatively weak. Conversely, the parameter of spatial propagation characteristics is relatively stronger and the parameter of temporal propagation characteristics is weaker. Actually, the development of communication technology is extended value of the constant $\mathrm{C}$. The level of technological spatial and temporal is higher, and the value of $\mathrm{C}$ is higher.

\section{The application multimedia communication technology with M2M solution}

M2M solution is more applied to multimedia communication process of electric power industry, transportation industry, safety and environmental protection industry and other industries. These industries have something in common, for example, pipelines, power device, oil wells need to remote monitoring facilities, which mainly using artificial patrol, this way exist some disadvantages such as poor real-time, high cost, waste of human resources, unable to carry on the monitoring to the harsh environment of the region and false positives may occur. Owning these disadvantages and improvement of the modernization level of industrial fields and multimedia communication technology, which makes the mode of production has been improved greatly: multimedia communication technology applying M2M can improve the ability to control, saving manpower and 
financial resources [4].

Because the application process of multimedia communication technology industrial is long and complex, leading to each M2M supplier has its advantages, it is difficult to form a unified standard and specification. For example, in the hardware aspect, M2M solution supplier has some characteristics, including technology homogenization serious, low added value, intense competition, each supplier has a proprietary protocol and standard; in the application system aspect, developers are lack of competition mechanism and scale of development, the development of the road will become narrower and narrower. The above defeats of M2M also lead to downstream telecom operators face the differentiating characteristics of various industries, in addition to do data channel has not found a new application services. Technical specification of non-uniform is another main reason for the rapid development of the business impact of M2M. Fast developing of market and the scale of the development of the market cannot do without the technical standards, interface and unified protocol enables terminal manufacturers in the standardization of products on the basis of greatly reduced development costs, to make the application of enterprise can choose all the terminal market, need not subject to any terminal manufacturers, to make the whole M2M solution in a normal state to enter the market.

Under the triple limitation of technology, business and policy, it is unrealistic to make different multimedia communication technology fulfill physical integration completely, because integration of the terminal media should have the ability to adapt to different channels. Under the influence of M2M, those terminal media which can access a variety of network called "multi-mode terminal media." Currently, the multi-mode terminal technology is mainly focused on the field of wireless communications which can be seamlessly connected to different types of wireless access networks, namely the connection between cellular UMITS/ EDGE/ GPRS and IEEE 802.11 WLAN [5]. However, compared with the development of multimedia communication technology abroad, our country's communication technology chain link has something missing, especially the important link in M2M service provider. This shows that, although the market application of China's M2M business has begun to take shape, but the industry is still relatively fragmented, the market is still in the exploratory stage, there is still a long way to go in the future.

\section{Multimedia communication technology development and collaborative development of M2M}

Practice of industry convergence in field of multimedia communication technology caused by M2M solution has led to traditional communication technology industry boundary fuzzy, and trend of multimedia communication technology convergence is obvious. Technological innovation of M2M is the driving force of multimedia communication technology convergence. Diffusion and application of M2M technology in multimedia communication technology triggered a spillover effect and lead to technology integration, while technology integration also eliminated the technological entry barriers of communication technology industry. The interaction of communication technology and M2M solution makes multimedia communication technology to form the same technological basis, since the traditional boundary between different communication technology industries tends to blur, or even disappear. Therefore, in the era of multimedia communication technology convergence promoted by $\mathrm{M} 2 \mathrm{M}$ solution, the communication technology is also undergoing convergence process like as other industries, showing the characteristics of industrial patterns of regularity development, becoming the inherent power sources which promoting the development of the whole multimedia communication technology industry.

The M2M solution is composed of five elements, which is tracking and management of the hardware, rocker survey, monitoring alarm, control, payment and transaction. In the short period, the common elements of the M2M business will guide the multimedia communication technology development. However, in the near future, many of elements of M2M solution need to unify and form a complete system of M2M platform, including the terminal access module, application access module, business treatment module, management module, the portal network interface module, monitoring interface module and so on. After the formation of M2M platform, a unified standard 
need to be set, this is a premise condition of a stable M2M market.

\section{Conclusion}

M2M has a unprecedented prospect, but if the puzzled problem including the communication terminal standardization, the integration of industrial chain and collaborative development of M2M, we are unable to realize the terminal cost reduction, unable to realize a large-scale business and unable to improve the M2M operation management and operation level, the final result is that we can not implement and extension M2M solution in the whole multimedia communication technology industry. We should summarize experience and shortage of domestic and international M2M development, carry out work of terminal standardization, module standardization, and interface standardization. In the future, we should construct a M2M management platform of unified and open architecture, integrate industry chain downstream parties to develop differentiation of value-added services, and enhance the value of the ARPU, which can meet the demand of multimedia communication technology, and personalized the M2M solution services for interests of all parties. Only in this way, can we effectively promote the development of multimedia communication technology, and format a profitable and benign M2M solution model.

\section{Literature References}

[1] H. F. Li: New Tendency of Network Communication Technology and Its Application. Shanghai Journalism Review. Vol. 08 (2006), p. 63-64.

[2] F. Williams: The Communications Revolution. Beverly Hills, Sage (1982), in Press.

[3] L. Wei, L. Q. Bao, T. J. Wu: The Evolution of Media Technology and Paradigm Transition of Communication Theory. Journalism \& Communication. Vol. 17 (2010). p. 18-21.

[4] Wu Geng, Johnsson, Himayat. M2M: From mobile to embedded Internet. Communication Magazine, IEEE, (2011). 36-43.

[5] L. Q. Bao, T. J. Wu: Communication Technology Route of the Media Integration. Modern Meida. Vol. 164 (2010). p. 151-152. 